\title{
Ginsenoside Rh2 inhibits HeLa cell energy metabolism and induces apoptosis by upregulating voltage-dependent anion channel 1
}

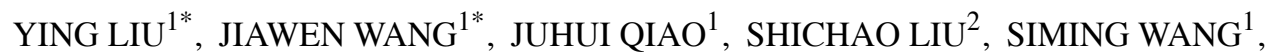 \\ DAQING ZHAO ${ }^{1}$, XUEYUAN BAI ${ }^{1}$ and MEICHEN LIU ${ }^{1}$ \\ ${ }^{1}$ Jilin Ginseng Academy and ${ }^{2}$ Academic Affairs Office, Changchun University of Chinese Medicine, \\ Changchun, Jilin 130117, P.R. China
}

Received March 7, 2020; Accepted July 27, 2020

DOI: $10.3892 /$ ijmm.2020.4725

\begin{abstract}
S)-Ginsenoside Rh2 [20(S)-GRh2], one of the main active components of Panax ginseng, induces apoptosis in a wide range of cancer cell types. The present study found that 20(S)-GRh2 reduces mitochondrial membrane potential, decreases adenosine triphosphate generation and induces reactive oxygen species in HeLa cervical cancer cells. In addition, 20(S)-GRh2 activated mitochondrion-dependent apoptosis and inhibited both mitochondrial oxidative phosphorylation and glycolysis in HeLa cells. It was found that voltage-dependent anion channel 1 (VDAC1) expression was significantly upregulated by 20(S)-GRh2 treatment, while hexokinase 2 expression was downregulated and segregated from the mitochondria. Furthermore, 20(S)-GRh2 promoted Bax transport from the cytoplasm to the mitochondria, and knockdown of VDAC1 inhibited Bax transport and apoptosis. These results suggest that VDAC1 is a novel target of $20(\mathrm{~S})-\mathrm{GRh} 2$. The present study provides a better understanding of the mechanistic link between cervical cancer metabolism and growth control, and these results may facilitate the development of new treatments for cervical cancer.
\end{abstract}

\section{Introduction}

Cervical cancer is the fourth most common gynecological malignant tumor that affects females, and results in $\sim 57,000$ new cases annually with 311,000 deaths worldwide $(1,2)$.

Correspondence to: Professor Xueyuan Bai or Dr Meichen Liu, Jilin Ginseng Academy, Changchun University of Chinese Medicine, 1035 Boshuo Road, Changchun, Jilin 130117, P.R. China

E-mail: baixy1212@163.com

E-mail: liumc0367@163.com

*Contributed equally

Key words: ginsenoside Rh2, apoptosis, mitochondrial metabolism, voltage-dependent anion channel 1, cervical cancer
In China, the incidence of cervical cancer has increased rapidly over the past 20 years (3). Surgery, radiotherapy and chemotherapy are currently the main treatment methods for cervical cancer (4). Although advances in these therapies have prolonged patient survival, the limitations of effective regimens are the resistance of tumor cells and their severe side effects (5). Thus, there is an urgent need for new anticancer drugs with fewer side effects that may improve survival rates.

Voltage-dependent anion channel (VDAC) family proteins in the mitochondrial outer membrane play an important role in cell survival and death (6). VDACs interact with members of the Bcl-2 family to participate in the release of cytochrome $c$ and the subsequent activation of caspase-9 (7,8). Furthermore, VDACs are involved in caspase-independent apoptosis (9). Among the three isomers (VDAC1, VDAC2 and VDAC3), VDAC1 shows the highest expression in mammalian mitochondria and plays an important role in apoptosis $(10,11)$. VDAC1 upregulation promotes apoptosis in multiple cell lines $(12,13)$. VDAC1 is also a target of various pro-apoptotic compounds such as curcumin, arsenic trioxide and cannabinoids (14-16).

A previous study showed that a VDAC1-based peptide induces apoptosis by releasing bound hexokinase 2 (HK2) from the mitochondria (17). HK2 is a crucial enzyme in the glycolytic pathway and antagonizes apoptosis through its direct interaction with VDAC1 (18). HK2 is overexpressed in a variety of tumor cell types, and HK2 is transported to the mitochondria and interacts with VDAC1, which promotes the development of anaerobic metabolism to compensate for the higher energy requirements in tumor cells (19). Most tumor cells still show active glucose uptake and glycolysis under aerobic conditions, a phenomenon known as the Warburg effect, and metabolic dysregulation affects tumor growth and cell death $(20,21)$. Several compounds, such as 3-bromopyruvate and methyl jasmonates, interact with HK2, causing HK2 to separate from mitochondria and trigger apoptosis $(22,23)$.

20(S)-Ginsenoside Rh2 [20(S)-GRh2], a protopanaxadiol-type ginsenoside extracted from Panax ginseng, is an active component with an anti-tumor effect that inhibits tumor cell growth and induces tumor cell apoptosis $(24,25)$. Previous studies showed that $20(\mathrm{~S})-\mathrm{GRh} 2$ has safety advantages (such as reverse multidrug resistance, balanced immunity and 
enhanced chemotherapy) (24) and shows no side effects, indicating 20(S)-GRh2 may be a promising new antitumor drug (26). Several reports demonstrated that 20(S)-GRh2 stimulates apoptosis and induces mitochondrial dysfunction by activating caspases, upregulating various pro-apoptotic proteins such as Bax and downregulating Bcl-XL (27-29). Therefore, 20(S)-GRh2 may stimulate HeLa cell apoptosis by affecting the mitochondria and regulating energy metabolism.

The presentstudy tested this hypothesis and the results showed that 20(S)-GRh2 exposure activated mitochondrion-dependent apoptosis and suppressed mitochondrial oxidative phosphorylation and glycolysis. To understand its underlying mechanism, it was found that VDAC1 was significantly upregulated in response to 20(S)-GRh2 treatment, while HK2 was downregulated and separated from mitochondria. Bax transport from the cytosol to the mitochondria was promoted by 20(S)-GRh2 and knockdown of VDAC1 reduced 20(S)-GRh2-induced Bax translocation and apoptosis. The results provided a more comprehensive understanding of the mechanistic association between cancer mitochondrial metabolism and tumor growth control by 20(S)-GRh2 and may facilitate the development of new therapeutic methods for cervical cancer.

\section{Materials and methods}

Materials and antibodies. 20(S)-GRh2 (98\% pure) was purchased from Shanghai Yanye Biotechnology Co., Ltd. The following primary antibodies were used in this study: Anti-HK2 rabbit polyclonal antibody (1:1,000; cat. no. 22029-1-AP; ProteinTech Group, Inc.), anti-VDAC1 rabbit monoclonal antibody (1:1,000; cat. no. AF1027; Beyotime Institute of Biotechnology), anti-prohibitin rabbit monoclonal antibody (1:1,000; cat. no. AF1126; Beyotime Institute of Biotechnology), anti-cytochrome $c$ rabbit monoclonal antibody (1:1,000; cat. no. AF2047; Beyotime Institute of Biotechnology), anti-Bax rabbit monoclonal antibody (1:1,000; cat. no. D2E11; Cell Signaling Technology, Inc.), anti-Bcl-2 rabbit polyclonal antibody (1:1,000; cat. no. abs131701, Absin) and anti- $\beta$-actin mouse monoclonal antibody $(1: 1,000$; cat. no. BS6007M; Bioworld Technology, Inc.).

Cell culture and transfection. HeLacells were obtained from the Cell Bank of the Chinese Academy of Sciences and cultured in DMEM (HyClone; Cytiva) with 10\% FBS (HyClone; Cytiva), $100 \mu \mathrm{g} / \mathrm{ml}$ penicillin and $100 \mu \mathrm{g} / \mathrm{ml}$ streptomycin (HyClone; Cytiva) at $37^{\circ} \mathrm{C}$ in a humidified atmosphere with $5 \% \mathrm{CO}_{2}$. Cells were transfected with a final concentration of $100 \mathrm{nM}$ small interfering RNA (siRNA) targeting VDACl using riboFECT CP reagent (Guangzhou Ribobio Co., Ltd.) according to the manufacturer's instructions. Following incubation for $24 \mathrm{~h}$, HeLa cells were treated with 20(S)-GRh2 (35 and $45 \mu \mathrm{M})$ for $24 \mathrm{~h}$ at $37^{\circ}$ before subsequent experimentation. The target sequence in negative controls for RNA interference is 5'-AAT TCTCCGAACGTGTCACGT-3', and the target sequences in VDAC1 for RNA interference were 5'-GGAGACCGCTGT CAATCTT-3' (siVC1-1) and 5'-GCTGCGACATGGATT TCGA-3' (siVC1-2).

Reverse transcription-quantitative PCR (RT-qPCR). Total RNA was extracted from HeLa cells treated with 20(S)-GRh2 using TRIzol ${ }^{\circledR}$ reagent (Invitrogen; Thermo Fisher Scientific, Inc.). RNA purity was measured using a BioSpec-nano spectrophotometer (Shimadzu Corporation), and total RNA was reverse transcribed using a PrimeScript RT Reagent Kit with gDNA Eraser (Takara Bio,Inc.) according to the manufacturer's instructions. RT-qPCR was performed using a SYBR-Green assay (Takara Bio, Inc.) on a CFX Connect Real-Time system (Bio-Rad Laboratories, Inc.). Fold-changes in the expression of each gene were calculated using the $2^{-\Delta \Delta C q}$ method (30). The following thermocycling conditions were used for the qPCR: Initial denaturation at $95^{\circ} \mathrm{C}$ for $30 \mathrm{sec}$, followed by 40 cycles at $95^{\circ} \mathrm{C}$ for $5 \mathrm{sec}$ and $60^{\circ} \mathrm{C}$ for $30 \mathrm{sec}$. GAPDH mRNA was used as an internal reference. The following primer pairs were used for the qPCR: GAPDH forward, 5'-CTGGGCTACACT GAGCACC-3' and reverse, 5'-AAGTGGTCGTTGAGGGCA ATG-3' (Harbin Xinhai Genetic Testing Co., Ltd.); VDAC1 forward, 5'-CTGACCTTCGATTCATCCTTCTC-3' and reverse, 5'-CTCCCGCTTGTACCCTGTC-3' (Harbin Xinhai Genetic Testing Co., Ltd.) and HK2 forward, 5'-AGCCACCAC TCACCCTAC-3' and reverse, 5'-CCATTGTCCGTTACTTTC AC-3' as well as forward, 5'-AGCCACCACTCACCCTAC-3' and reverse, 5'-CCCATTGTCCGTTACTTTC-3' (Comate Bioscience Co., Ltd.).

Cell viability assessment. The Cell Counting Kit-8 (CCK-8) assay (Wuhan Boster Biological Technology Co., Ltd.) was used to assess cell viability according to the manufacturer's instructions. HeLa cells were treated with 20 (S)-GRh2 at $37^{\circ} \mathrm{C}$ for $24 \mathrm{~h}$ at various concentrations $(35,45,55$ and $65 \mu \mathrm{M})$ to determine the $\mathrm{IC}_{50}$ values. In addition, cells were treated with $\mathrm{IC}_{50}$ concentrations at $37^{\circ} \mathrm{C}$ at various timepoints $(6,12,24$, 36 and $48 \mathrm{~h}$ ). Following treatment, $20 \mu \mathrm{l} \mathrm{CCK-8} \mathrm{solution} \mathrm{was}$ added to each well, and the plate was incubated at $37^{\circ} \mathrm{C}$ for $1 \mathrm{~h}$. Absorption at a wavelength of $450 \mathrm{~nm}$ was recorded using an Infinite M200 pro reader (Tecan Group, Ltd.) and the cell viability was calculated.

Cell apoptosis analysis. Cells were treated with various concentrations (35 and $45 \mu \mathrm{M}$ ) of $20(\mathrm{~S})-\mathrm{GRh} 2$ at $37^{\circ} \mathrm{C}$ for $24 \mathrm{~h}$. Cells were then suspended in 1X Binding Buffer and incubated with $5 \mu \mathrm{l}$ Annexin V-FITC and $5 \mu \mathrm{l}$ PI (BD Biosciences) at room temperature in the dark for $15 \mathrm{~min}$. The cells were analyzed with a Flow Sight flow cytometer (Amnis Corporation) and data were analyzed using IDEAS software v6.1 (Amnis Corporation). Apoptotic cells were also analyzed by Hoechst 33342 staining (Beyotime). Briefly, following 20(S)-GRh2 (35 and $45 \mu \mathrm{M})$ treatment, cells were incubated with Hoechst 33342 at $37^{\circ} \mathrm{C}$ in the dark for $30 \mathrm{~min}$. Cells treated with $45 \mu \mathrm{M}$ DMSO waere used as the control group. Images were captured under an EVOS FL Auto fluorescence microscope (Thermo Fisher Scientific, Inc.) at x295 magnification.

Determination of the mitochondrial membrane potential $(M M P)$. MMP alterations were measured using a JC-1 MMP Assay kit (Beijing Solarbio Science \& Technology Co., Ltd.). HeLa cells were treated with 20(S)-GRh2 (35 and $45 \mu \mathrm{M})$ at $37^{\circ} \mathrm{C}$ for $24 \mathrm{~h}$ and then washed with cold PBS. A total of $10 \mu \mathrm{M}$ carbonyl cyanide 3-chlorophenylhydrazone (CCCP) was used to induce a decrease in mitochondrial membrane potential for 
20 min as a positive control. JC-1 working solution was added to the cell culture medium, and cells were incubated at $37^{\circ} \mathrm{C}$ in the dark for $20 \mathrm{~min}$. Cells were imaged using an EVOS FL Auto fluorescence microscope (Thermo Fisher Scientific, Inc.) at $\mathrm{x} 295$ magnification.

MMP was also measured using the cationic dye rhodamine 123 (Beyotime Institute of Biotechnology). Briefly, HeLa cells were pre-treated with $20(\mathrm{~S})-\mathrm{GRh} 2(35$ and $45 \mu \mathrm{M})$ at $37^{\circ} \mathrm{C}$ for $24 \mathrm{~h}$ and then incubated with $2 \mu \mathrm{M}$ rhodamine 123 at $37^{\circ} \mathrm{C}$ in the dark for $30 \mathrm{~min}$. Fluorescence intensity was detected using the Flow Sight flow cytometer (Amnis Corporation) and data were analyzed using IDEAS software v6.1 (Amnis Corporation).

Western blotting. Cells treated with 20(S)-GRh2 (35 and $45 \mu \mathrm{M}$ ) at $37^{\circ} \mathrm{C}$ for $24 \mathrm{~h}$ were lysed in RIPA buffer (Beyotime Institute of Biotechnology) pre-cooled at $4^{\circ} \mathrm{C}$. Mitochondrial and cytoplasmic proteins were extracted using a cell mitochondrial isolation kit (Beyotime Institute of Biotechnology). Protein quantification was performed using the BCA method (Beyotime Institute of Biotechnology). Equal amounts of protein $(30 \mu \mathrm{g})$ were subjected to $12 \%$ SDS-PAGE and then transferred onto a nitrocellulose membrane (Pall Corporation). The membranes were blocked with $5 \%$ skim milk powder and PBS for $1 \mathrm{~h}$ at room temperature, then incubated with primary antibodies overnight at $4^{\circ} \mathrm{C}$, followed by incubation with goat polyclonal secondary antibody to mouse IgG-H\&L (HRP) (1:1,000; cat. no. 115-005-00; Jackson ImmunoResearch Laboratories, Inc.) and goat anti-rabbit IgG H\&L (HRP) (1:1,000; cat. no. 111-005-003; Jackson ImmunoResearch Laboratories, Inc.) for $1 \mathrm{~h}$ at $37^{\circ} \mathrm{C}$. Development was performed with an ECL kit (Beyotime Institute of Biotechnology). Imaging and densitometric analysis were performed using an iBright FL1000 imaging system (Invitrogen; Thermo Fisher Scientific, Inc.). $\beta$-actin and prohibitin were used as loading controls.

Measurement of cellular ATP levels. Total ATP levels were determined using a Chemiluminescence ATP assay kit (Beyotime Institute of Biotechnology) according to the manufacturer's instructions. Briefly, the culture solution was removed, followed by addition of $200 \mu 1$ lysate to each well of a six-well plate and centrifuged at $12,000 \mathrm{x}$ g to collect the cell supernatant. In addition, $100 \mu \mathrm{l}$ ATP detection working solution was added to the 96-well assay plates and incubated at room temperature for $3 \mathrm{~min}$. Subsequently, $20 \mu \mathrm{l}$ sample was added to the wells and analyzed on the Infinite M200 pro reader (Tecan Group, Ltd.). Meanwhile, added gradient diluions of ATP standard solution were added to 96-well plates to generate a standard curve, and ATP concentration was calculated according to the standard curve.

Determination of reactive oxygen species (ROS) levels. To detect intracellular ROS levels, the ROS-sensitive probe 2',7'-dichlorodihydrofluorescein diacetate $\left(\mathrm{H}_{2} \mathrm{DCFDA}\right.$; Beyotime Institute of Biotechnology) was used. Cells were treated with the indicated concentrations of $20(\mathrm{~S})-\mathrm{GRh} 2$ (35 and $45 \mu \mathrm{M}$ ) for $24 \mathrm{~h}$ and then incubated with $10 \mu \mathrm{M}$ $\mathrm{H}_{2}$ DCFDA in the dark for $30 \mathrm{~min}$ at $37^{\circ} \mathrm{C}$. The fluorescence intensity of the cells was measured with a Flow Sight flow cytometer (Amnis Corporation).
Mitochondrial oxidative phosphorylation (OXPHOS) and glycolysis assays. A total of $5 \times 10^{3} \mathrm{HeLa}$ cells were seeded into the B-G pores of an XF 8 Cell Culture Microplate (Agilent Technologies, Inc.) in $80 \mu \mathrm{l}$ medium. Equal volumes of media were then added into the $\mathrm{A}$ and $\mathrm{H}$ pores, followed by overnight incubation at $37^{\circ} \mathrm{C}$ with $5 \% \mathrm{CO}_{2}$. Only the calibration solution was left on both sides of the culture chamber. HeLa cells were treated with 20(S)-GRh2 (35 and $45 \mu \mathrm{M}$ ) for $24 \mathrm{~h}$. The probe plate of the Seahorse XFp sensor was incubated overnight in the calibration solution the day before the experiment. Cells were washed with $180 \mu \mathrm{l}$ DMEM without bicarbonate and then incubated at $37^{\circ} \mathrm{C}$ for $1 \mathrm{~h}$ before measurement.

Oxygen consumption rates (OCR) and extracellular acidification rates (ECAR) were measured using an XFp Extracellular Flux Analyzer (Agilent Technologies, Inc.) according to manufacturer's instructions. The OCR was continuously recorded for 12 cycles, with each cycle consisting of 3 min of mixing and 3 min of measurement. Basal respiration was measured during cycles 1-3. Further measurement of OCR was run as follows: As 4-6 cycles of oligomycin injection (oligo, ATP synthase inhibitor, $5 \mu \mathrm{M}$ ), 7-9 cycles of carbonyl cyanide 4-(trifluoromethoxy) phenylhydrazone (FCCP, proton, $5 \mu \mathrm{M}$ ) injection and 10-12 cycles of rotenone/antimycin A (AA/Rot, complex I and III inhibitor, $2.5 \mu \mathrm{M}$ ) injection [all, XFp Cell Mito Stress Test kit (Agilent Technologies, Inc.)]. Further measurement of ECAR was run as follows: 4-6 cycles of glucose (substrate for hexokinase, $10 \mathrm{mM}$ ) injection, 7-9 cycles of oligomycin (oligo, ATP synthase inhibitor, $5 \mu \mathrm{M}$ ) injection and 10-12 cycles of 2-Deoxy-D-glucose (2-DG, competitive inhibitor of hexokinase, $50 \mathrm{mM}$ ) injection. The use of these inhibitors permits the determination of key aspects of mitochondrial function (31).

Caspase activity assays. Assays for caspase-3 (cat. no. C1115), -8 (cat. no. C1151) and -9 (cat. no. C1157) activities were performed using kits according to the manufacturer's instructions (Beyotime Institute of Biotechnology). Briefly, cells were washed with PBS, collected by centrifugation $(600 \mathrm{x} \mathrm{g}$, $5 \mathrm{~min}$ ) at $4^{\circ} \mathrm{C}$ and resuspended in ice cold lysis buffer. A total of $100 \mu 1$ lysate was used per $2 \times 10^{6}$ cells and incubated on ice for $20 \mathrm{~min}$. The protein concentration in the supernatant was measured with a Bradford assay kit (Tiangen Biotech Co., Ltd.). Subsequently, $50 \mu 1$ cell lysate supernatant was mixed with $10 \mu 1$ Ac-DEVD-pNA (2 mM) for caspase-3, Ac-IETD-pNA (2 mM) for caspase- 8 and Ac-LEHD-pNA ( $2 \mathrm{mM}$ ) for caspase-9 in $40 \mu \mathrm{l}$ assay buffer for $4 \mathrm{~h}$ at $37^{\circ} \mathrm{C}$, and then analyzed with an Infinite M200 pro reader (Tecan Group, Ltd.).

Statistical analysis. Statistical analyses were performed using GraphPad Prism software 6 (GraphPad Software, Inc.). In quantitative analyses shown in histograms, values were obtained from three independent experiments and expressed as the mean \pm SD. One-way ANOVA followed by Dunnett's post-hoc test was used to compare between two groups. $\mathrm{P}<0.05$ was considered to indicate a statistically significant difference.

\section{Results}

20(S)-GRh2 inhibits cell viability and induces apoptosis in HeLa cells. The CCK-8 method was used to examine the effect of 20(S)-GRh2 (Fig. 1A) on HeLa cell viability. Cells 

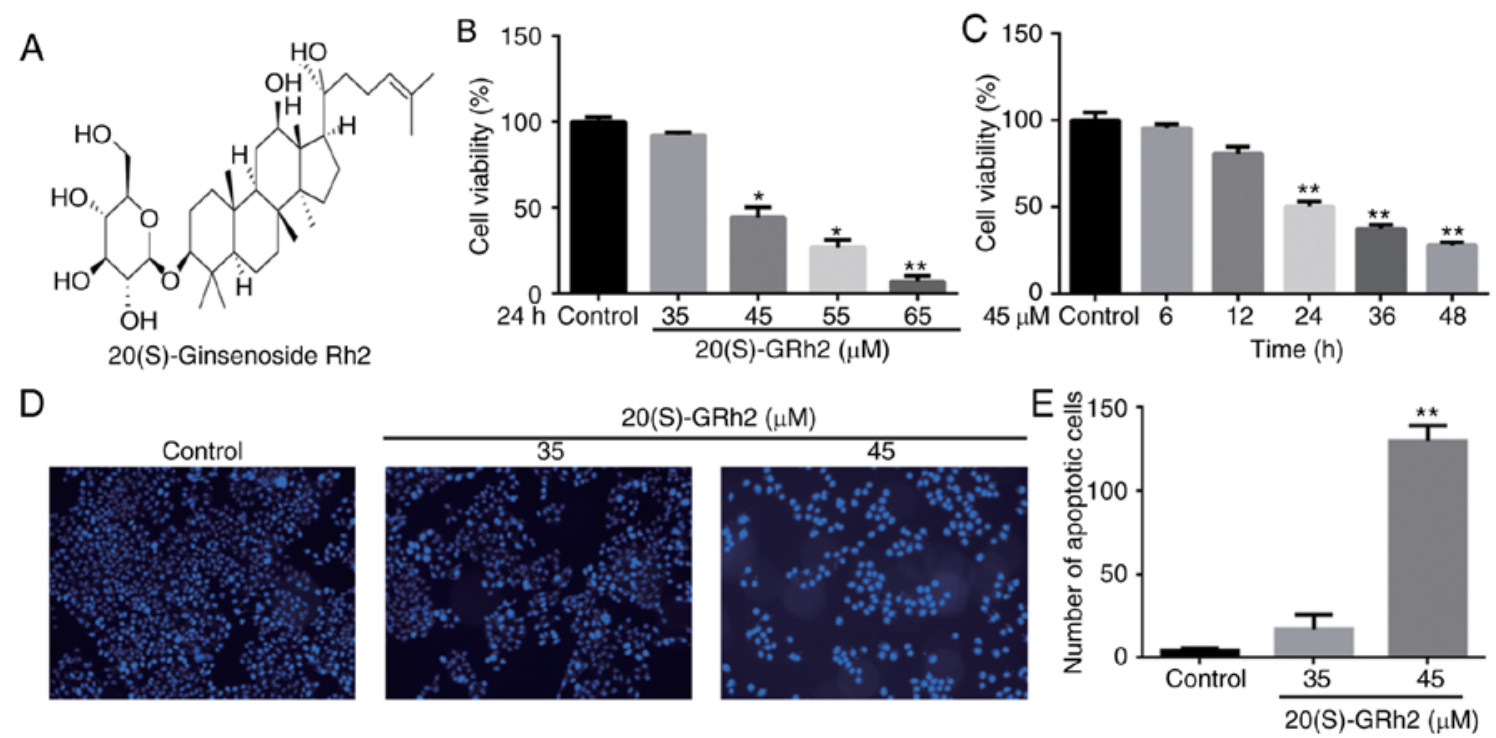

$\mathrm{F}$
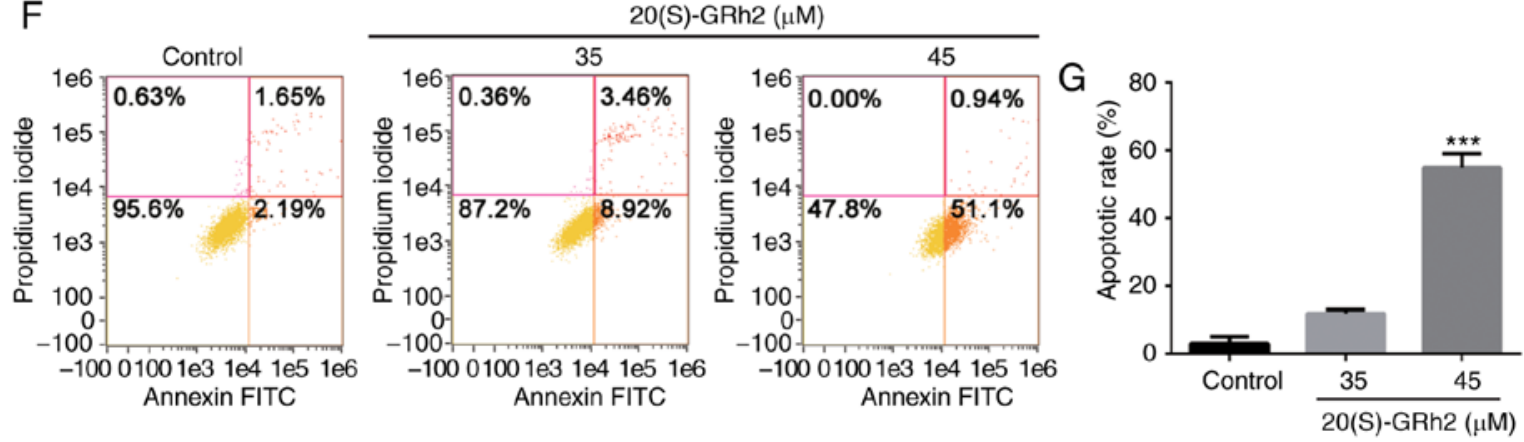

Figure 1. 20(S)-GRh2 inhibits cell viability and induces apoptosis in HeLa cells. (A) The structural formula of 20(S)-GRh2. Hela cells were treated with (B) various 20 (S)-GRh 2 concentrations for $24 \mathrm{~h}$ or (C) $45 \mu \mathrm{M} 20$ (S)-GRh2 at different time intervals compared with the control group (0 h). Cell viability was detected using Cell Counting Kit-8 assays. Data are expressed as a percentage of the control, which was set at 100\%. (D) Representative photomicrographs and (E) quantified results of Hoechst staining in the control group and various concentrations of 20(S)-GRh2 for 24 h. Magnification, x295. (F and G) HeLa cells were treated with various concentrations of 20 (S)-GRh2 for $24 \mathrm{~h}$. The percentage of apoptotic cells was measured by flow cytometry using annexin V-FITC/PI staining. All results were counted from three independent experiments. $n=3$. Data were analyzed using one-way ANOVA and presented as the mean \pm standard deviation. ${ }^{*} \mathrm{P}<0.05 ;{ }^{* *} \mathrm{P}<0.01$ and ${ }^{* * *} \mathrm{P}<0.001$ vs. control. 20(S)-GRh2, 20(S)-ginsenoside Rh2.

were treated with various doses of 20(S)-GRh2 for different timepoints. The results showed that 20(S)-GRh2 reduced the viability of HeLa cells in dose- and time-dependent manners (Fig. 1B and C). The $\mathrm{IC}_{50}$ of 20(S)-GRh2 was calculated to be $\sim 45 \mu \mathrm{M}$, and 35 and $45 \mu \mathrm{M}$ for further experiments.

It was next examined whether 20(S)-GRh2 inhibited cell viability via inducing apoptosis. Hoechst staining showed an increase in the amount of condensed and fragmented nuclei in cells treated with $45 \mu \mathrm{M} 20(\mathrm{~S})$-GRh2 compared with the control group (Fig. 1D and E), indicating that 20(S)-GRh2 induced apoptosis in HeLa cells. Annexin V and PI double staining was used to detect the apoptotic rate of HeLa cells treated with 20(S)-GRh2. The percentages of apoptotic cells upon treatment with 0,35 and $45 \mu \mathrm{M} 20$ (S)-GRh2 were $3.84,12.38$ and $52.04 \%$, respectively (Fig. $1 \mathrm{~F}$ and G). Taken together, these findings suggested that $20(\mathrm{~S})$-GRh2 reduces cell viability by inducing apoptosis in HeLa cells.

20(S)-GRh2 reduces MMP, decreases ATP generation and induces ROS in HeLa cells. Mitochondria play a central role in the growth and apoptosis of cells (32). Therefore, it was next assessed whether 20(S)-GRh2-induced apoptosis was associated with mitochondrial dysfunction. The effects of
20(S)-GRh2 on the MMP, an indicator of mitochondrial functions, were assessed using JC-1 and rhodamine-123 staining. Fluorescence microscopy showed that cells exposed to 20(S)-GRh2 showed increased conversion of JC-1 aggregates (red) to JC-1 monomers (green). Following treatment with $10 \mu \mathrm{M} \mathrm{CCCP}$ as the positive control, the cells showed obvious green fluorescence, indicating that $10 \mu \mathrm{M}$ CCCP almost completely depleted the MMP of HeLa cells (Fig. 2A). Flow cytometry showed that $45 \mu \mathrm{M} 20$ (S)-GRh2 treatment significantly reduced the fluorescence intensity of rhodamine-123 (Fig. 2B). MMP and ATP generation are indicators of mitochondrial function (33), and thus the effects of 20(S)-GRh2 on the cellular ATP levels were assessed. Compared with the control group, 20(S)-GRh2 exposure reduced cellular ATP levels in HeLa cells by $14 \%(35 \mu \mathrm{M})$ and $49 \%(45 \mu \mathrm{M})$ (Fig. 2C). The decrease in MMP and ATP levels showed that 20(S)-GRh2 induced mitochondrial dysfunction in HeLa cells.

It was hypothesized that 20(S)-GRh2-induced mitochondrial dysfunction may cause increased levels of ROS. To test this hypothesis, ROS levels in 20(S)-GRh2-treated HeLa cells were measured. Low concentrations of 20(S)-GRh2 (35 $\mu \mathrm{M})$ had no significant effect on cellular ROS, whereas 

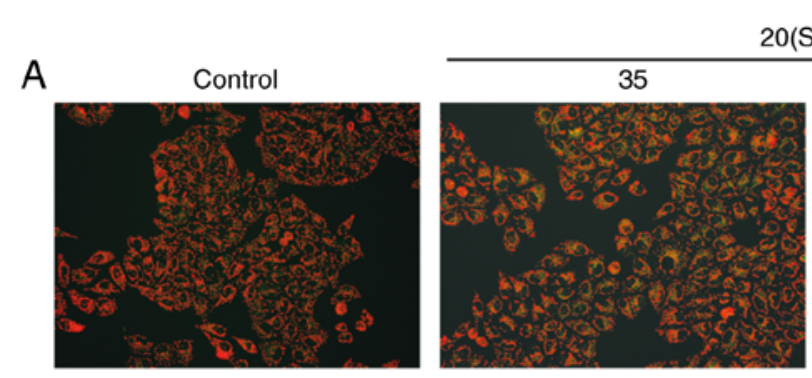

O(S)-GRh2 $(\mu \mathrm{M})$
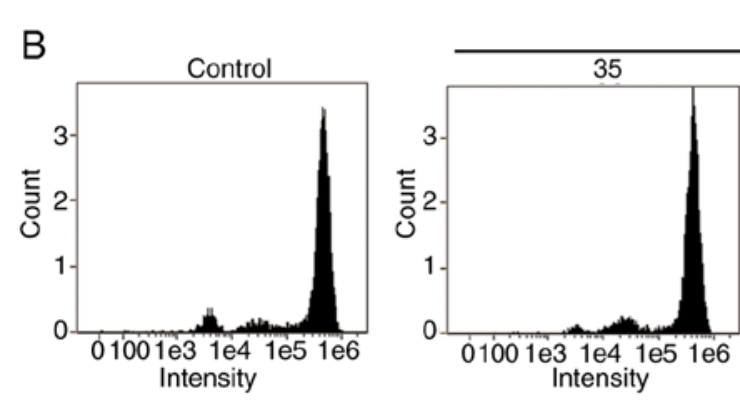

20(S)-GRh2 $(\mu \mathrm{M})$
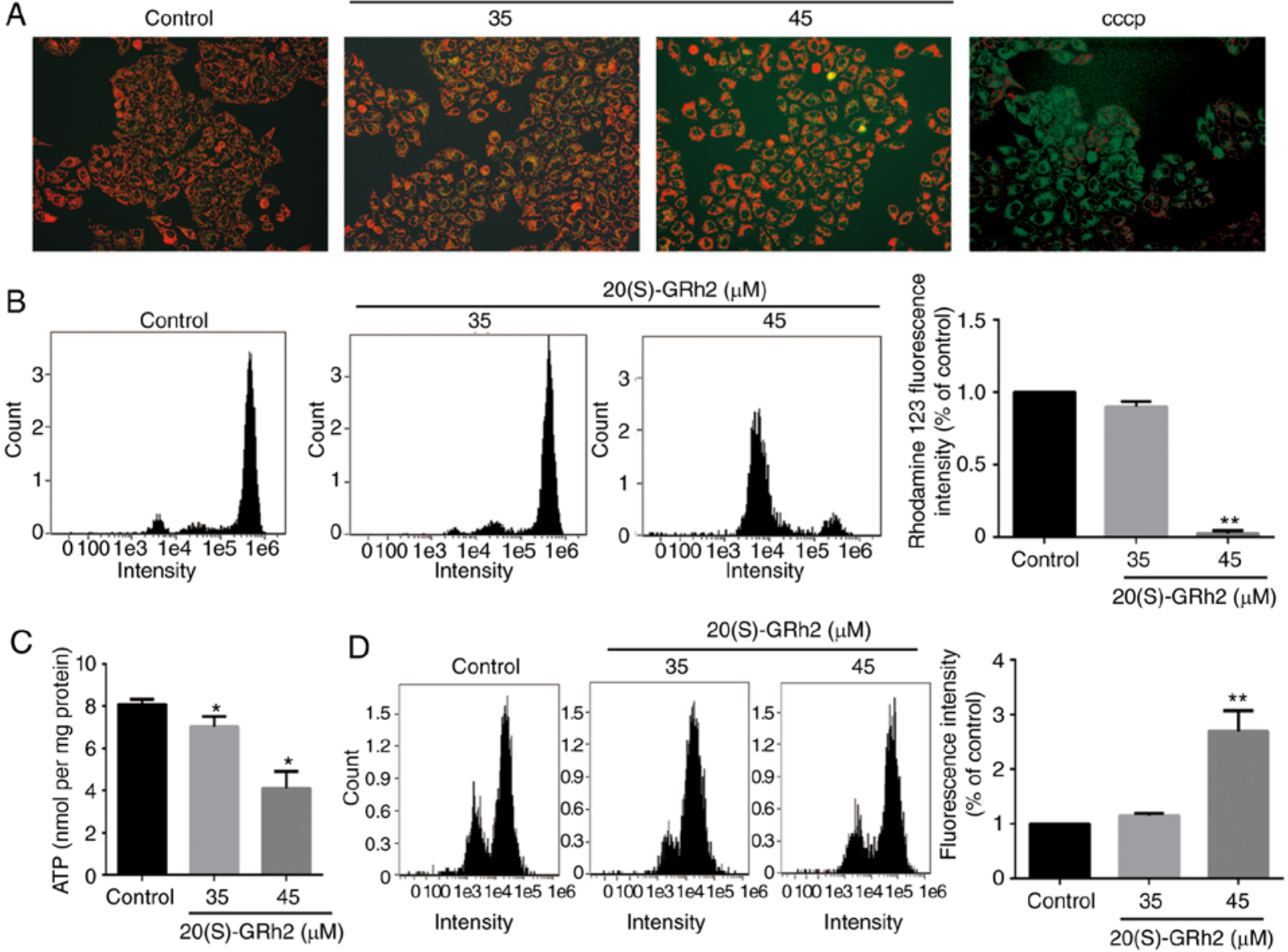

20(S)-GRh2 $(\mu \mathrm{M})$
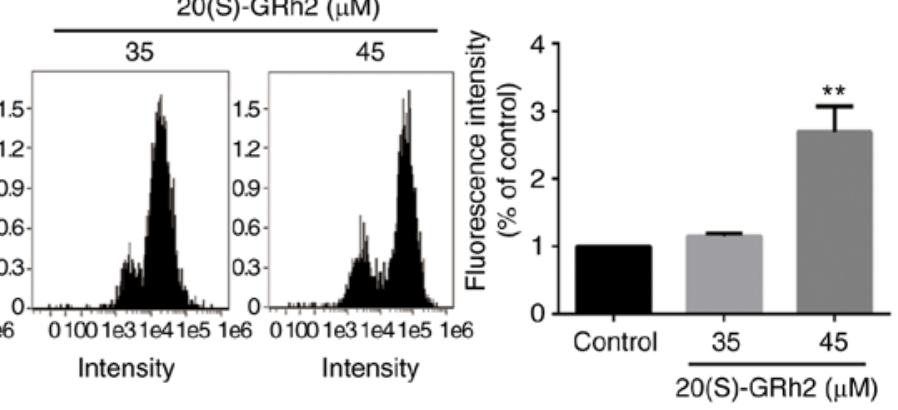

Figure 2. 20(S)-GRh2-induced apoptosis is associated with mitochondrial dysfunction. (A) HeLa cells were treated with various concentrations of 20(S)-GRh2 for $24 \mathrm{~h}$. The mitochondrial membrane potential was measured by JC-1 staining and detected using a fluorescence microscope. Magnification, x295. (B) HeLa cells were incubated with 35 or $45 \mu \mathrm{M} 20$ (S)-GRh2 for $24 \mathrm{~h}$ and rhodamine 123 dye for $30 \mathrm{~min}$. Fluorescence emission was measured using flow cytometry and quantified. (C) ATP levels were detected using a chemiluminescence ATP assay kit. (D) 2',7'-Dichlorodihydrofluorescein diacetate was applied to assess ROS levels. The results were counted from three independent experiments. $n=3$. Data were analyzed using one-way ANOVA and presented as the mean \pm standard deviation. ${ }^{*} \mathrm{P}<0.05$ and ${ }^{* *} \mathrm{P}<0.01$ vs. control. 20(S)-GRh2, 20(S)-ginsenoside Rh2.

a high concentration of $20(\mathrm{~S})$-GRh2 $(45 \mu \mathrm{M})$ significantly increased cellular ROS by 2.69 -fold compared with control cells (Fig. 2D).

20(S)-GRh2 inhibits mitochondrial OXPHOS and glycolysis. Since the mitochondrial electron transfer chain is the main target site of ROS (34), the present study hypothesized that 20(S)-GRh2 might affect mitochondrial ATP generation, which is involved in 20(S)-GRh2-induced cell death. The XFp extracellular flux analyzer was then used to assess the role of 20(S)-GRh2 on mitochondrial OXPHOS and glycolysis, the two major energy metabolism pathways, in real-time. OXPHOS was measured by quantifying the OCR of the medium and glycolysis was assessed by quantifying the ECAR of the medium. Treatment with 20(S)-GRh2 (35 and $45 \mu \mathrm{M})$ caused a decrease in the OCR and altered cellular responses to typical mitochondrial complexes inhibitors (including oligomycin, an ATP synthase inhibitor; FCCP, a mitochondrial uncoupler and AA/Rot, inhibitors of complex I and III), suggesting that the mitochondrial OXPHOS capacity was inhibited (Fig. 3A). HeLa cells had a significantly reduced OCR linked to ATP production and maximum respiratory capacity following 20(S)-GRh2 (35 and $45 \mu \mathrm{M}$ ) treatment (Fig. 3B). However, in
HeLa cells treated with 20(S)-GRh2, the OCR associated with non-mitochondrial respiration was also reduced (Fig. 3A). This result indicated that 20(S)-GRh2 may also inhibit the oxygen consumption of the non-mitochondrial respiratory pathway, but the exact molecular mechanism was unclear.

Numerous cancer cells break down glucose by aerobic glycolysis to obtain the energy needed for growth in a process known as the Warburg effect (35). It was next examined whether 20(S)-GRh2 also induced this effect on glycolysis in HeLa cells. The results showed that both basal and stimulated ECAR reduced after $45 \mu \mathrm{M} 20$ (S)-GRh2 treatment (Fig. 3C). 20(S)-GRh2 $(45 \mu \mathrm{M})$ significantly reduced the ECAR linked to glycolytic capacity and the glycolytic reserve in HeLa cells (Fig. 3D). At $35 \mu \mathrm{M}, 20$ (S)-GRh2 had no effect on ECAR (Fig. 3C) but inhibited OCR (Fig. 3A) and decreased cellular ATP levels (Figs. 2C and 3B), suggesting that 20(S)-GRh2 decreased ATP production primarily by inhibition of OXPHOS.

Mitochondrial pathway is involved in 20(S)-GRh2-induced HeLa cell apoptosis. To further assess the apoptotic process induced by 20(S)-GRh2, the activation of caspase-3, a critical marker of apoptosis, was investigated. As shown in Fig. 4A, 
A

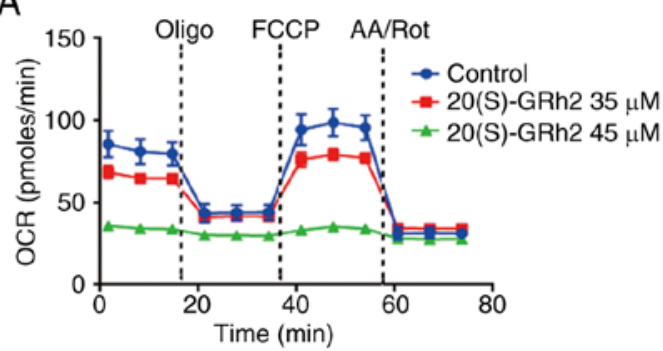

$\mathrm{C}$

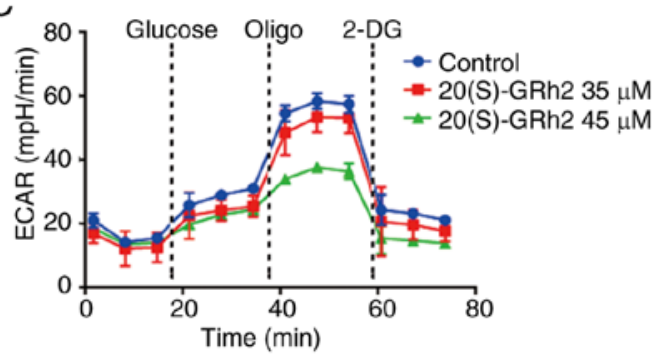

B

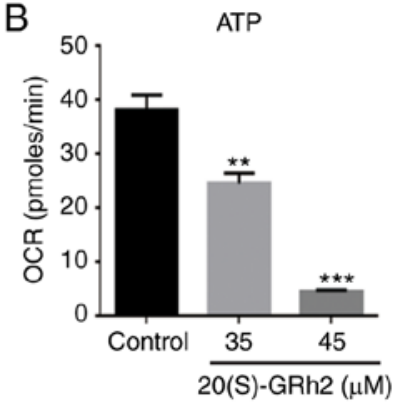

D

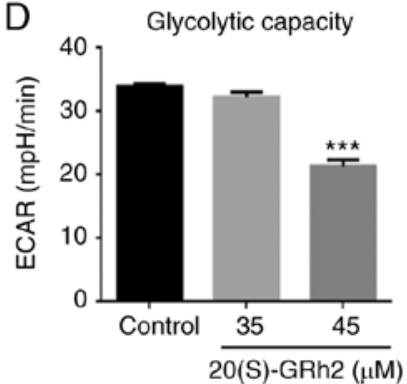

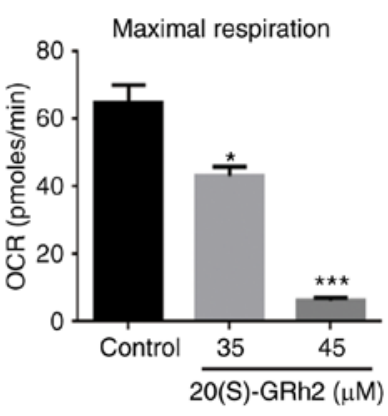

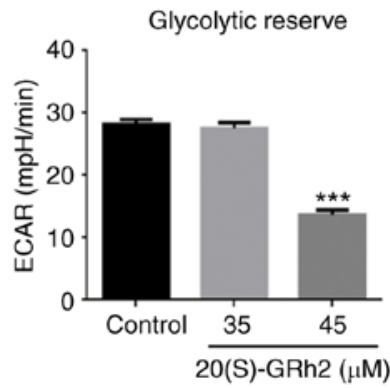

Figure 3. Effects of 20(S)-GRh2 on mitochondrial respiration and glycolysis. HeLa cells were treated with 20 (S)-GRh2 at indicated concentrations for $24 \mathrm{~h}$ and the dynamics of OCR and ECAR were measured. (A) Graphical representation of the OCR measurement over time; sequential additions are indicated as: Oligo, FCCP and AA/Rot. (B) The effects of 20(S)-GRh2 on the ATP-linked OCR and maximal respiration capacity-linked OCR calculated from the OCR curves. Data analyzed using one-way ANOVA and presented as the mean \pm standard deviation. $\mathrm{n}=3$. ${ }^{*} \mathrm{P}<0.05 ;{ }^{* *} \mathrm{P}<0.01$ and ${ }^{* * *} \mathrm{P}<0.001$ vs. control. (C) Graphical representation of the ECAR measurement over time; sequential additions are indicated as: Glucose, Oligo and 2-DG. (D) The effects of 20(S)-GRh2 on the glycolytic capacity-linked ECAR and glycolytic reserve-linked ECAR calculated from the ECAR curves. Data were analyzed using one-way ANOVA and presented as the mean \pm standard deviation. $n=3$. ${ }^{* * *} \mathrm{P}<0.001$ vs. control. 20(S)-GRh2, 20(S)-ginsenoside Rh2; OCR, oxygen consumption rate; ECAR, extracellular acidification rate; Oligo, oligomycin; FCCP, carbonyl cyanide 4-(trifluoromethoxy) phenylhydrazone; AA/Rot, antimycin A/rotenone; 2-DG, 2-Deoxy-D-glucose; $\mathrm{mpH} / \mathrm{min}$, the rate of $\mathrm{mpH}$ change per minute.

20(S)-GRh2 treatment significantly increased caspase-3 activity in a dose-dependent manner. Next, the upstream regulators of caspase-3 involved in 20(S)-GRh2-induced apoptosis were determined. Mitochondrion-associated caspase-9 activity was significantly increased by $45 \mu \mathrm{M} 20$ (S)-GRh2 but did not affect death receptor-associated caspase-8 activity in HeLa cells (Fig. 4B and C). Since caspase-9 is activated by Bcl-2 family proteins (36), it was investigated whether 20(S)-GRh2 affected the expression of Bcl-2 and Bax proteins. $45 \mu \mathrm{M}$ 20(S)-GRh2 induced Bax upregulation and Bcl-2 downregulation (Fig. 4D). Bcl-2 and Bax induces the release of cytochrome $c$ from the mitochondria into the cytoplasm (37). Western blot analysis showed that 20 (S)-GRh2 significantly promoted the release of cytochrome $c$ into the cytoplasm, which coincided with caspase-9 activation (Fig. 4E). Once cytochrome $c$ is released into the cytoplasm, it activates caspase-9, triggering a cascade causing mitochondrion-mediated apoptosis (38); hence, the results indicated that $20(\mathrm{~S})-\mathrm{GRh} 2$ induces apoptosis via the mitochondrial pathway (Fig. 4E).

Upregulation of VDAC1 plays a role in 20(S)-GRh2-induced apoptosis. VDAC1 plays an important role in regulating mitochondrial energy metabolism and mitochondrion-mediated apoptosis (39). Therefore, the role of VDAC1 in 20(S)-GRh2-induced apoptosis was assessed. VDAC1 expression in HeLa cells was significantly increased by $45 \mu \mathrm{M}$ 20(S)-GRh2 treatment (Fig. 5A and B), indicating that induction of VDAC1 may be involved in 20(S)-GRh2-mediated induction of apoptosis in HeLa cells. To determine the role of VDAC1 in HeLa cell apoptosis induced by 20(S)-GRh2, a synthetic siRNA targeting VDAC1 to knockdown VDAC1 in HeLa cells was used (Fig. 5C and D). Annexin V staining showed that 20(S)-GRh2-induced apoptosis was suppressed in VDAC1-silenced cells (Fig. 5E and F). These results showed that 20(S)-GRh2 induced apoptosis in HeLa cells by regulating VDAC1.

20(S)-GRh2-induced reduction of HK2 results in VDAC1 upregulation. To further elucidate the molecular mechanism underlying 20(S)-GRh2-induced VDAC1 upregulation on HeLa cell apoptosis, known VDAC1-binding proteins were investigated. HK2 interacts with VDAC1 and plays major roles in tumor cell metabolism, including mitochondrial ATP synthesis and glucose metabolism (40). HK2 also inhibits tumor cell apoptosis by inhibiting changes in mitochondrial membrane permeability (41). Therefore, HK2 expression was measured. It was found that the expression of mitochondrial HK2 and cytosolic HK2 in HeLa cells treated with $45 \mu \mathrm{M}$ 20(S)-GRh2 was decreased which was consistent with the pattern of total HK2 (Fig. 6A-C). By contrast, the expression levels of VDAC1 protein was increased in response to $45 \mu \mathrm{M}$ 20(S)-GRh2 (Fig. 6D), indicating that 20(S)-GRh2 induces changes in the expression levels of HK2 and VDAC1 that are closely related to its induction of apoptosis.

Bax expression is involved in 20(S)-GRh2-induced apoptosis. It was found that 20(S)-GRh2 treatment induced Bax upregulation and promoted Bax translocation to the mitochondria (Fig. 7A and B). Bax expression was significantly suppressed when VDAC1 was silenced by siRNA (Fig. 7C and D). The 
A

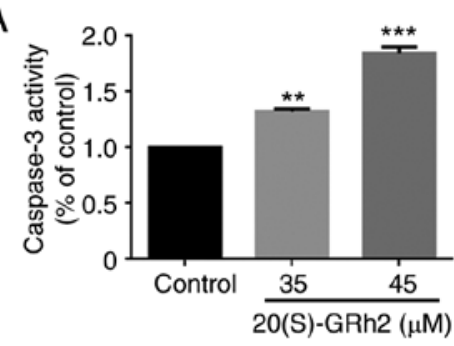

$\mathrm{B}$

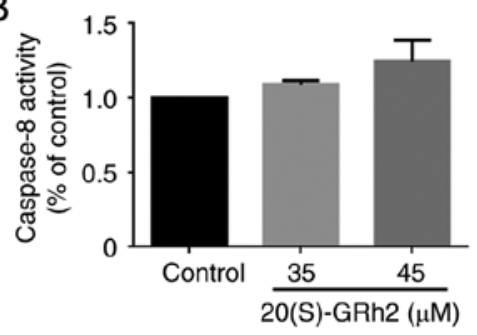

C

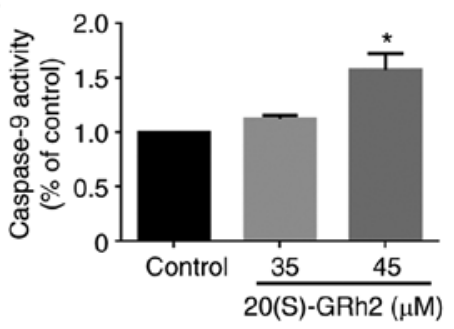

D
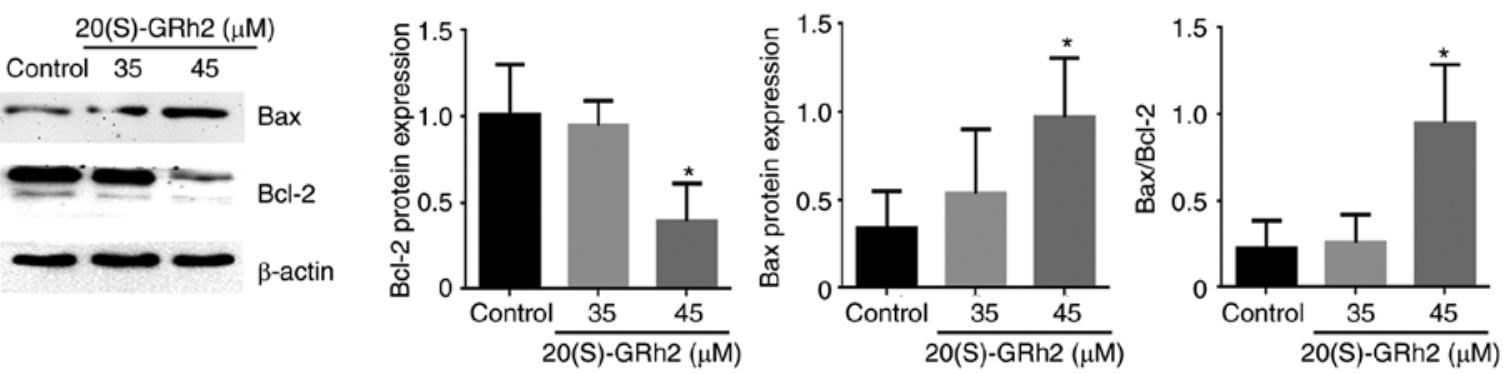

E
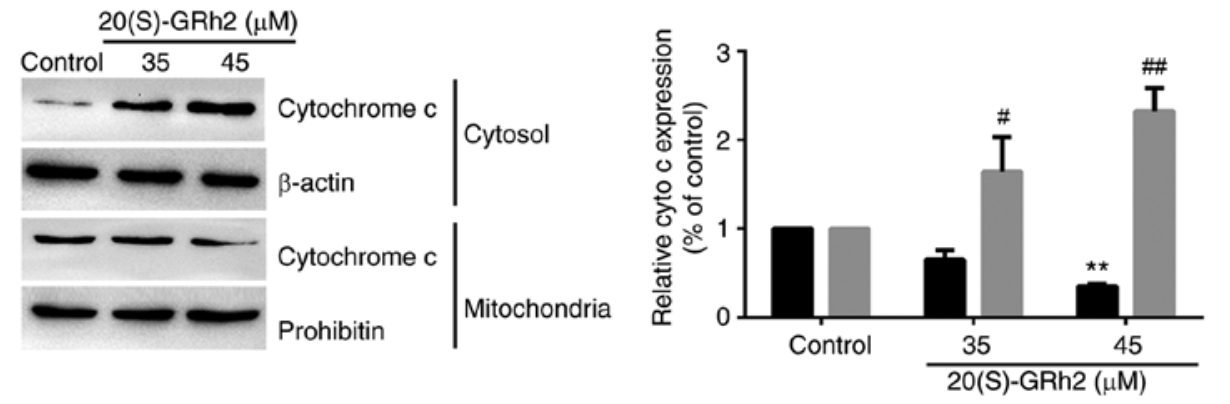

Figure 4. 20(S)-GRh2 induces HeLa cell apoptosis via the mitochondrial pathway. HeLa cells were treated with 20(S)-GRh2 at indicated concentrations for 24 h. (A) Caspase-3, (B) caspase- 8 and (C) caspase-9 activity were separately detected using a caspase activity assay kit. Data were analyzed using one-way ANOVA and presented as the mean \pm standard deviation. $\mathrm{n}=3$. ${ }^{*} \mathrm{P}<0.05 ;{ }^{* *} \mathrm{P}<0.01$ and ${ }^{* * * *} \mathrm{P}<0.001$ vs. control. (D) The protein expression levels of $\mathrm{Bcl}-2$ and Bax in HeLa cells were detected by western blot analysis. Data were analyzed using one-way ANOVA and presented as the mean \pm standard deviation. $\mathrm{n}=3$. ${ }^{*} \mathrm{P}<0.05$ vs. control. (E) Western blot analysis to detect cytochrome c levels in mitochondrial and cytosolic fractions of HeLa cells. Data analyzed using one-way ANOVA and presented as the mean \pm standard deviation. $\mathrm{n}=3$. ${ }^{* *} \mathrm{P}<0.01$ vs. control (mitochondrial fraction). ${ }^{\#} \mathrm{P}<0.05$ and ${ }^{\# \#} \mathrm{P}<0.01$ vs. control (cytosolic fraction). 20(S)-GRh2, 20(S)-ginsenoside Rh2.

aforementioned results showed that the separation of HK2 from the mitochondria promoted the translocation of Bax to mitochondria and the release of cytochrome $c$ into the cytoplasm.

We hypothesize that treatment of HeLa cells with 20(S)-GRh2 causes metabolic stress leading to upregulation of VDAC1, the translocation of Bax to the mitochondria and subsequent apoptosis of cancer cells (Fig. 8).

\section{Discussion}

The present study found that 20(S)-GRh2, a pharmacologically active component of Panax ginseng, exerted significant cytotoxicity in HeLa cells. Exposure to 20(S)-GRh2 altered mitochondrial function in HeLa cells, leading to mitochondrion-related apoptosis, confirming that 20(S)-GRh2 induces apoptosis of HeLa cells, as reported previously $(25,42)$. In addition, cell death induced by 20 (S)-GRh2 was associated with the regulation of energy metabolism. Treatment with 20(S)-GRh2 significantly inhibited OXPHOS and glycolysis. The dual targeting of mitochondrial and glycolysis pathways by 20 (S)-GRh2 may explain why ROS generation, MMP collapse and ATP reduction were observed in HeLa cells treated with 20(S)-GRh2.
In addition, the OCR that corresponds to non-mitochondrial $\mathrm{O}_{2}$ consumption was also slightly affected by 20(S)-GRh2. Mitochondria consume $>95 \%$ of the $\mathrm{O}_{2}$ of aerobic higher organisms; however, $10 \%$ of cellular oxygen uptake in mammal cells is due to non-mitochondrial respiration (43). It has been proposed that an increase in non-mitochondrial oxygen consumption might serve as a protective mechanism to remove ROS when it is present at potentially harmful concentrations (44). This suggestion provides a plausible explanation for the decrease in non-mitochondrial oxygen consumption observed in the present study, as it would help increase in ROS generation and oxidative stress to further induce apoptosis.

The mechanism by which $20(\mathrm{~S})-\mathrm{GRh} 2$ regulates cell death and energy metabolism was next elucidated. 20(S)-GRh2 influenced the protein levels of VDAC1 and HK2, which are important regulators of glucose metabolism $(45,46)$, and the changes in VDAC1 and HK2 protein levels were inversely associated. Treatment with 20(S)-GRh2 inhibited mitochondrial metabolism, leading to dissociation of HK2 from mitochondrial VDAC1, which regulated the transfer of Bax to the mitochondria and allowed cytochrome $c$ to enter the cytosol via the outer mitochondrial membrane. These events led to apoptosis induced by metabolic stress. A loss 
A

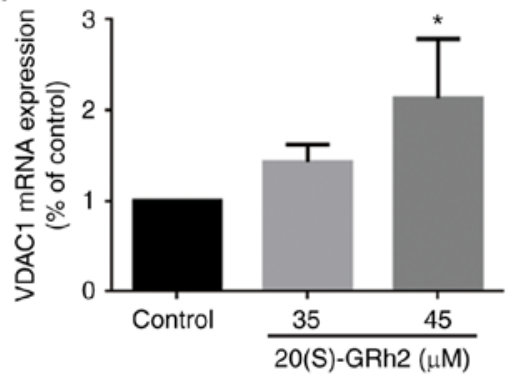

C

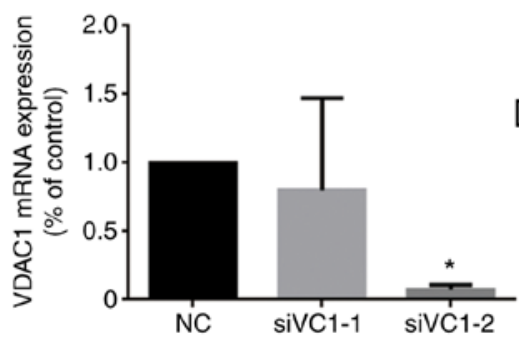

F

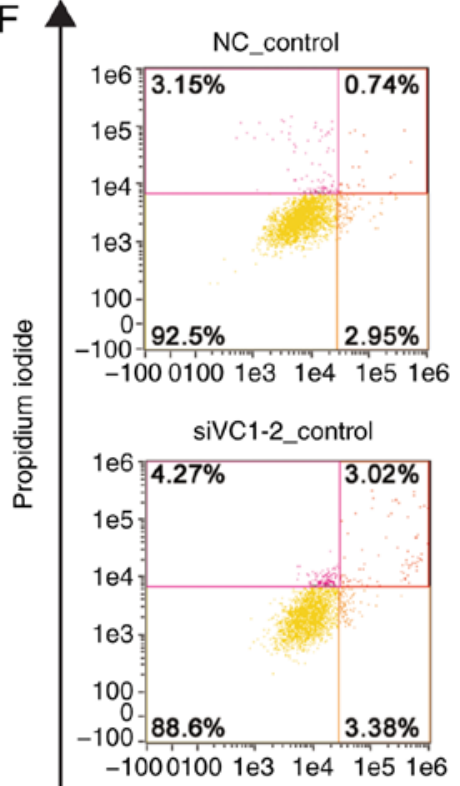

B

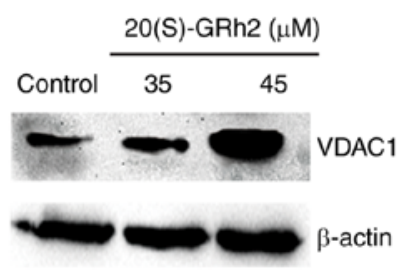

D

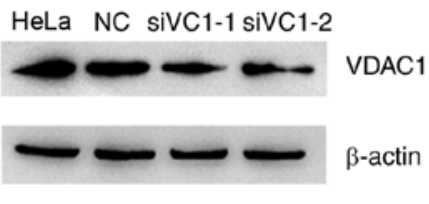

20(S)-GRh2 $(\mu \mathrm{M})$

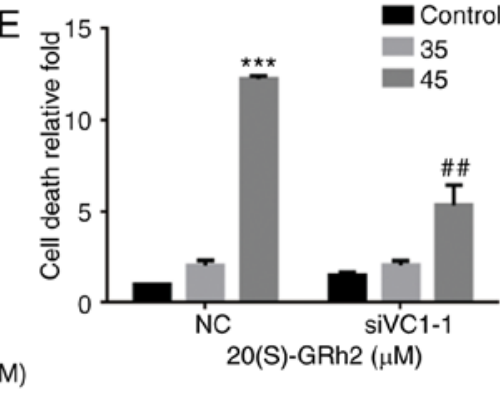

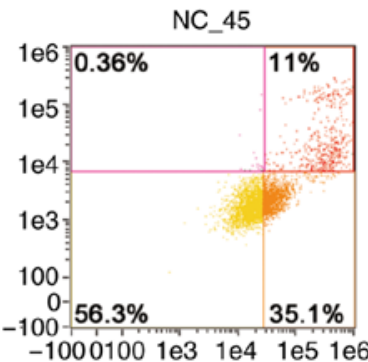

-1000100 e3 1e4 1e5 1e

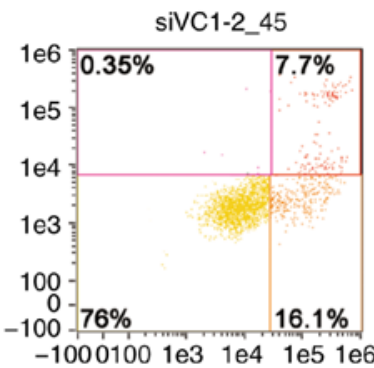

Annexin FITC

Figure 5. Upregulation of VDAC1 plays a role in 20(S)-GRh2-induced apoptosis. (A) RT-qPCR was performed to examine VDAC1 expression in HeLa cells. Data were analyzed using one-way ANOVA and presented as the mean \pm standard deviation. $n=3$. ${ }^{*} \mathrm{P}<0.05$ vs. control. (B) Western blot was applied to assess VDAC1 protein expression. Data were analyzed using one-way ANOVA and presented as the mean \pm standard deviation. $\mathrm{n}=3$. ${ }^{* * *} \mathrm{P}<0.001$ vs. control. (C) VDAC1 expression levels in cells transfected with NC, siVC1-1 or siVC1-2 was assessed by RT-qPCR. Data were analyzed using one-way ANOVA and presented as the mean \pm standard deviation. $n=3 .{ }^{*} \mathrm{P}<0.05$ vs. NC. (D) VDAC1 protein expression levels in cells transfected with NC, siVC1-1 or siVC1-2 was assessed by western blotting. (E and F) Flow cytometry was performed to assess the apoptosis of HeLa cells transfected with NC or siVC1-2. Data analyzed using one-way ANOVA and presented as the mean \pm standard deviation. $\mathrm{n}=3$. ${ }^{* * *} \mathrm{P}<0.001$ vs. NC control; ${ }^{\# \#} \mathrm{P}<0.01$ vs. siVC1-2 control. RT-qPCR, reverse transcription-quantitative PCR; 20(S)-GRh2, 20(S)-ginsenoside Rh2; VDAC1, voltage-dependent anion channel 1; siVC1, small interfering RNA targeting VDAC1; NC, negative control.

of the MMP during 20(S)-GRh2-induced apoptosis and the opening of mitochondrial permeability transition pores were observed. The present findings are the first to show that this mechanism is associated with $20(\mathrm{~S})$-GRh2-induced apoptosis. These results also showed that VDAC1 may be a target in the process of HeLa cell apoptosis induced by 20(S)-GRh2.

By transferring HK2 to the mitochondria, an acidic anti-hypoxic microenvironment suitable for tumor cell survival can be formed and maintained (47). The interaction between $\mathrm{HK} 2$ and VDAC1 provides metabolic preponderance for cancer cells by enhancing anaerobic glycolysis, which is called the Warburg effect (48). When HK2 separates from the mitochondria, cells become sensitive to many apoptotic factors (49). Many anticancer compounds that target the mitochondria have been shown to release HK2 from the mitochondria (50-52). The present study found that $20(\mathrm{~S})-\mathrm{GRh} 2$ disaggregated HK2 from the mitochondria in HeLa cells. 
A
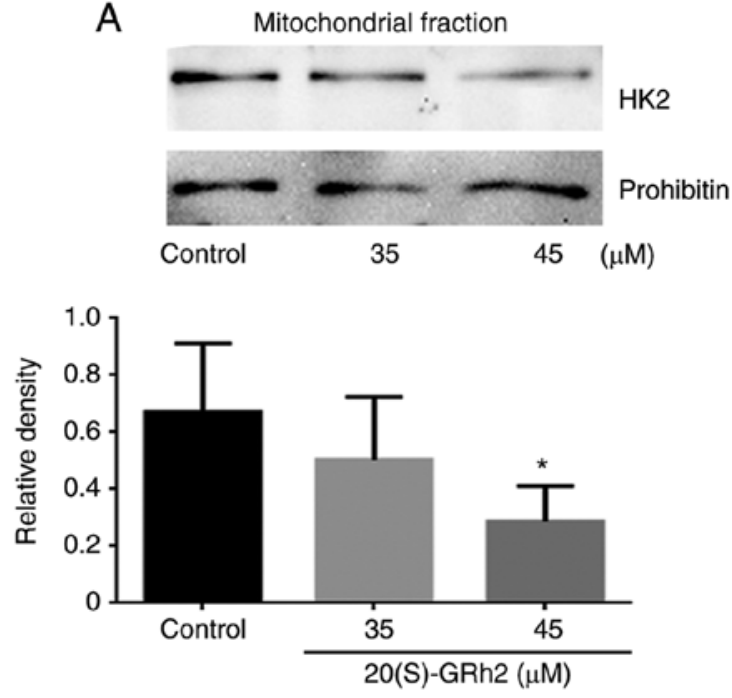

C
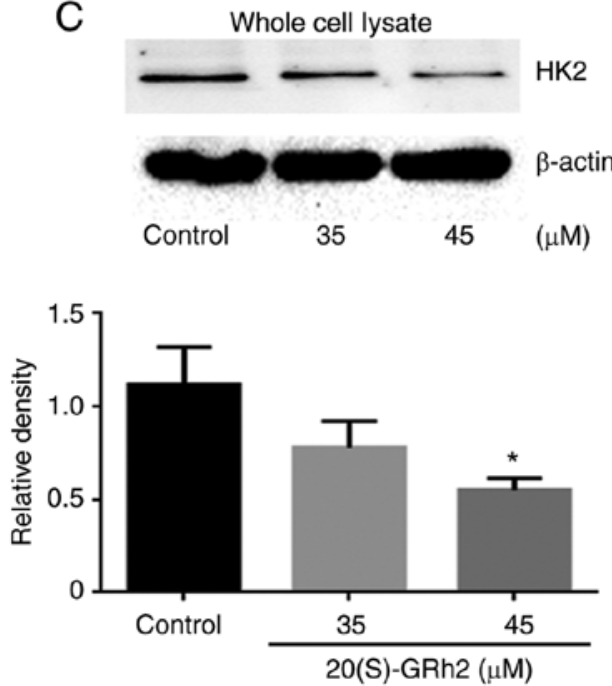

B
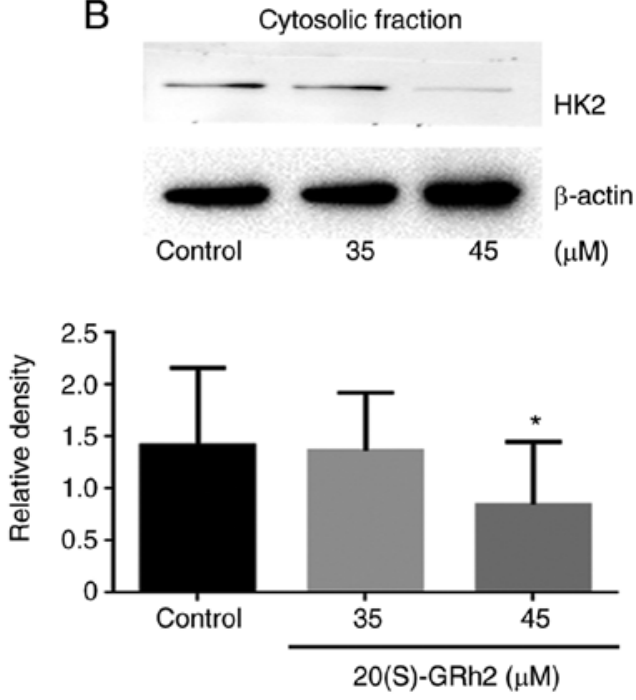

D
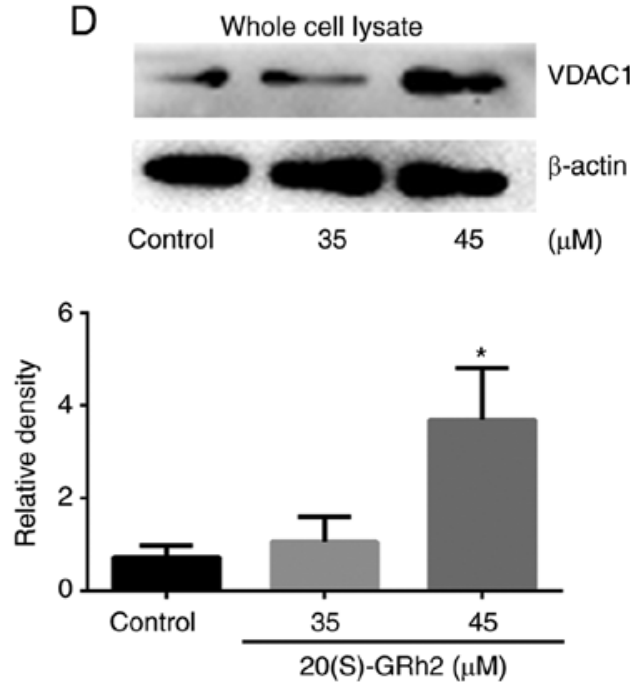

Figure 6. 20(S)-GRh2-induced reduction of HK2 results in VDAC1 upregulation. (A) Mitochondrial and (B) cytosolic HK2 expression was determined using western blotting. HeLa cell lysates were analyzed by western blotting with antibodies for (C) HK2 and (D) VDAC1. Data were analyzed using one-way ANOVA and presented as the mean \pm standard deviation. $n=3$. ${ }^{*} \mathrm{P}<0.05$ vs. control. 20(S)-GRh2, 20(S)-ginsenoside Rh2; VDAC1, voltage-dependent anion channel 1 ; HK2, hexokinase 2.

However, whether desorption of HK2 was due solely to the direct interaction of $20(\mathrm{~S})-\mathrm{GRh} 2$ with VDAC1 was unclear, and an interaction between $20(\mathrm{~S})-\mathrm{GRh} 2$ and HK2 cannot be ruled out. Thus, 20(S)-GRh2-induced cell death is a cumulative effect of VDAC1 blockade and desorption of HK2 from mitochondria. ROS production, MMP collapse and ATP decrease may be the result of VDAC shutdown. The wide range of antitumor activities of 20(S)-GRh2 includes inhibiting tumor growth, restraining tumor progression and strengthening chemotherapeutic responses (24). Previous studies have shown that 20 (S)-GRh2 inhibits the growth of many tumor cell types and its potential mechanism has been confirmed. Ginsenoside $\mathrm{Rh} 2$ also inhibited proliferation and promotes apoptosis of H1299 cells by inducing endoplasmic reticulum stress mediated by ROS (53). The synergistic effect of autophagy and $\beta$-catenin signal transduction inhibited the proliferation and migration of human liver cancer HepG2, Hep3B and Huh7 cells, and restrained tumor growth in HepG2-xenografted mice $(54,55)$. Ginsenoside Rh2 inhibited cell proliferation, promoted apoptosis and reversed the resistance of colon cancer to the chemotherapeutic drug oxaliplatin (56). The p53 tumor suppressor is also activated in response to ginsenosides $(25,57)$. Ginsenoside Rh2 also induced apoptosis of HCT-116 and SW-480 cells via p53 activation (58).

In conclusion, the present findings have revealed VDAC1 as a new target of $20(\mathrm{~S})-\mathrm{GRh} 2$. These results have provided further understanding of the metabolic mechanism of ginsenoside Rh2 against cancer and have validated the potential clinical value for $20(\mathrm{~S})-\mathrm{GRh} 2$ or anticancer drugs based on 20(S)-GRh2 in cervical cancer in vitro. However, the present study only studied a human papillomavirus (HPV)-positive cervical cancer cell. Based on evidence suggesting the heterogeneity in cervical cancer-related pathways in terms of HPV status, we are currently examining the effects of 20(S)-GRh2 in a comprehensive panel of human cervical cancer-derived cell lines, including HPV-positive and HPV-negative cell lines. Future research will examine the efficacy of 20(S)-GRh2 in an in vivo system. 


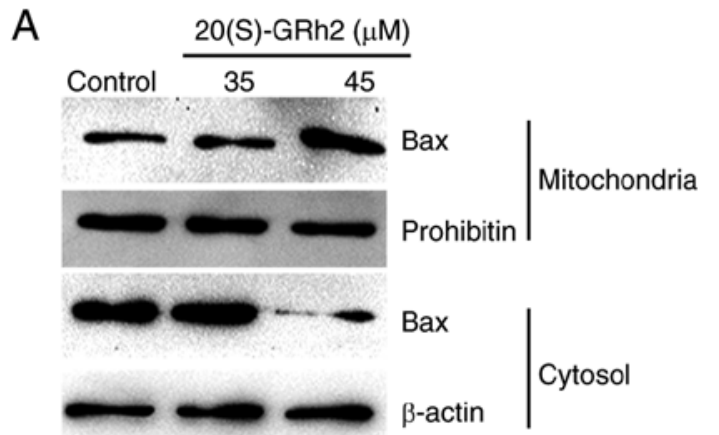

B

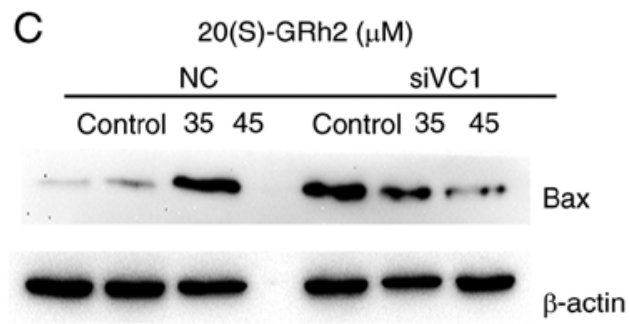

Whole cell lysate

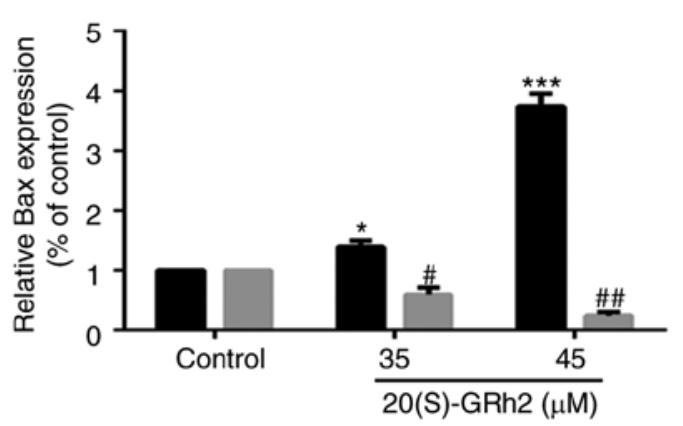

Mitochondria Cytosol

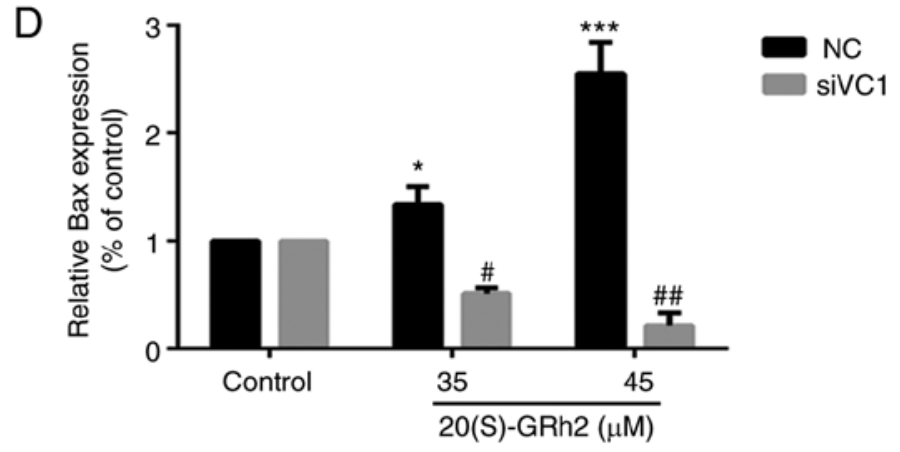

Figure 7. Mitochondrial translocation of Bax is involved in VDAC1-mediated apoptosis, which is induced by 20(S)-GRh2. (A and B) Subcellular localization of Bax was examined by western blotting. Prohibitin acted as the loading control for mitochondrial samples; $\beta$-actin acted as the loading control for cytosolic fractions. Data analyzed using one-way ANOVA and presented as the mean \pm standard deviation. $n=3$. ${ }^{*} \mathrm{P}<0.05$ and ${ }^{* * *} \mathrm{P}<0.001$ vs. control (mitochondrial fraction). ${ }^{\#} \mathrm{P}<0.05$ and ${ }^{\# \#} \mathrm{P}<0.01$ vs. control (cytosol fraction). (C and D) Western blot analysis showed that Bax expression is downregulated in VDAC1-silenced HeLa cells. Data were analyzed using one-way ANOVA and presented as the mean \pm standard deviation. $\mathrm{n}=3 .{ }^{*} \mathrm{P}<0.05$ and ${ }^{* * *} \mathrm{P}<0.001$ vs. NC control. ${ }^{*} \mathrm{P}<0.05$ and ${ }^{\# \#} \mathrm{P}<0.01$ vs. siVC1 control. 20(S)-GRh2, 20(S)-ginsenoside Rh2; siVC1, small interfering RNA targeting voltage-dependent anion channel 1; NC, negative control.

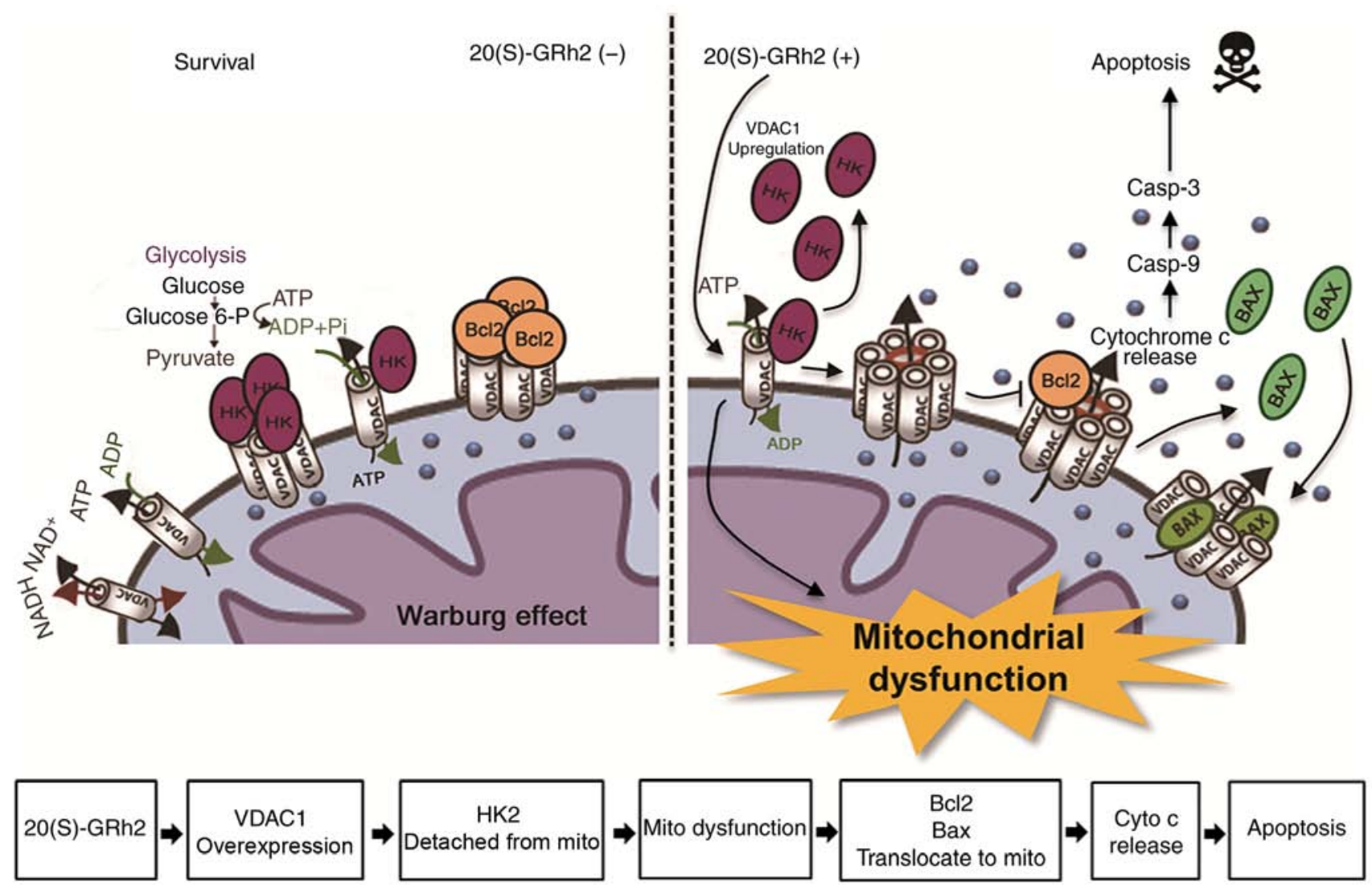

Figure 8. 20(S)-GRh2 causes metabolic stress leading to upregulation of VDAC1, translocation of Bax to mitochondria and subsequent apoptosis of HeLa cells. 20(S)-GRh2, 20(S)-ginsenoside Rh2; VDAC1, voltage-dependent anion channel 1; HK2, hexokinase 2; mito, mitochondria; casp, caspase. 


\section{Acknowledgements}

Not applicable.

\section{Funding}

This work was supported by the National Key Research and Development program of China (grant no. 2017YFC1702104) and grants from the National Natural Foundation of China (grant no. 81703663); and Key Project At Central Government Level: The Ability Establishment of Sustainable Use For Valuable Chinese Medicine Resources (grant no. 2060302).

\section{Availability of data and materials}

The datasets used and/or analyzed during the current study are available from the corresponding author on reasonable request.

\section{Authors' contributions}

ML and JW managed data collection and designed the experiments. YL, JQ, SL and SW performed the experiments. YL and ML wrote the manuscript. DZ and XB contributed to data analysis, supervised the experiments and helped draft the manuscript. All authors read and approved the final manuscript.

\section{Ethics approval and consent to participate}

Not applicable.

\section{Patient consent for publication}

Not applicable.

\section{Competing interests}

The authors declare that they have no competing interests.

\section{References}

1. Chen S and Wang J: HAND2-AS1 inhibits invasion and metastasis of cervical cancer cells via microRNA-330-5p-mediated LDOC1. Cancer Cell Int 19: 353, 2019.

2. Arbyn M, Weiderpass E, Bruni L, de Sanjosé S, Saraiya M, Ferlay $\mathrm{J}$ and Bray F: Estimates of incidence and mortality of cervical cancer in 2018: A worldwide analysis. Lancet Glob Health 8: e191-e203, 2020.

3. Di J, Rutherford S and Chu C: Review of the cervical cancer burden and population-based cervical cancer screening in China. Asian Pac J Cancer Prev 16: 7401-7407, 2015.

4. Iwata T, Miyauchi A, Suga Y, Nishio H, Nakamura M, Ohno A, Hirao N, Morisada T, Tanaka K, Ueyama H, et al: Neoadjuvant chemotherapy for locally advanced cervical cancer. Chin J Cancer Res 28: 235-240, 2016.

5. Marchetti C, Fagotti A, Tombolini V, Scambia G and De Felice F Survival and toxicity in neoadjuvant chemotherapy plus surgery versus definitive chemoradiotherapy for cervical cancer: A systematic review and meta-analysis. Cancer Treat Rev 83: $101945,2020$.

6. Shoshan-Barmatz V, Ben-Hail D, Admoni L, Krelin Y and Tripathi SS: The mitochondrial voltage-dependent anion channel 1 in tumor cells. Biochim Biophys Acta 1848: 2547-2575, 2015.

7. Abu-Hamad S, Zaid H, Israelson A, Nahon E and Shoshan-Barmatz V: Hexokinase-I protection against apoptotic cell death is mediated via interaction with the voltage-dependent anion channel-1: Mapping the site of binding. J Biol Chem 283: $13482-13490,2008$
8. Shoshan-Barmatz V, Israelson A, Brdiczka D and Sheu SS: The voltage-dependent anion channel (VDAC): Function in intracellular signalling, cell life and cell death. Curr Pharm Des 12: 2249-2270, 2006.

9. Adachi M, Higuchi H, Miura S, Azuma T, Inokuchi S, Saito H, Kato $\mathrm{S}$ and Ishii $\mathrm{H}$ : Bax interacts with the voltage-dependent anion channel and mediates ethanol-induced apoptosis in rat hepatocytes. Am J Physiol Gastrointest Liver Physiol 287: G695-G705, 2004.

10. Zaid H, Abu-Hamad S, Israelson A, Nathan I and ShoshanBarmatz V: The voltage-dependent anion channel-1 modulates apoptotic cell death. Cell Death Differ 12: 751-760, 2005.

11. Yamamoto T, Yamada A, Watanabe M, Yoshimura Y, YamazakiN, Yoshimura Y, Yamauchi T, Kataoka M, Nagata T, Terada H and Shinohara Y: VDAC1, having a shorter N-terminus than VDAC2 but showing the same migration in an SDS-polyacrylamide gel, is the predominant form expressed in mitochondria of various tissues. J Proteome Res 5: 3336-3344, 2006.

12. Li B, Chen X, Yang W, He J, He K, Xia Z, Zhang J and Xiang G: Single-walled carbon nanohorn aggregates promotes mitochondrial dysfunction-induced apoptosis in hepatoblastoma cells by targeting SIRT3. Int J Oncol 53: 1129-1137, 2018.

13. Nagakannan P,Islam MI,Karimi-Abdolrezaee S and EftekharpourE: Inhibition of VDAC1 protects against glutamate-induced oxytosis and mitochondrial fragmentation in hippocampal HT22 cells. Cell Mol Neurobiol 39: 73-85, 2019.

14. Tewari D, Ahmed T, Chirasani VR, Singh PK, Maji SK, Senapati S and Bera AK: Modulation of the mitochondrial voltage dependent anion channel (VDAC) by curcumin. Biochim Biophys Acta 1848: 151-158, 2015.

15. Watanabe M, Funakoshi T, Unuma K, Aki T and Uemura K: Activation of the ubiquitin-proteasome system against arsenic trioxide cardiotoxicity involves ubiquitin ligase Parkin for mitochondrial homeostasis. Toxicology 322: 43-50, 2014.

16. Rimmerman N, Ben-Hail D, Porat Z, Juknat A, Kozela E, Daniels MP, Connelly PS, Leishman E, Bradshaw HB, Shoshan-Barmatz V and Vogel Z: Direct modulation of the outer mitochondrial membrane channel, voltage-dependent anion channel 1 (VDAC1) by cannabidiol: A novel mechanism for cannabinoid-induced cell death. Cell Death Dis 4: e949, 2013.

17. Liu Y, Cheng H, Zhou Y, Zhu Y, Bian R, Chen Y, Li C, Ma Q, Zheng Q, Zhang Y, et al: Myostatin induces mitochondrial metabolic alteration and typical apoptosis in cancer cells. Cell Death Dis 4: e494, 2013.

18. Pastorino JG and Hoek JB: Regulation of hexokinase binding to VDAC. J Bioenerg Biomembr 40: 171-182, 2008.

19. Dolder M, Wendt S and Wallimann T: Mitochondrial creatine kinase in contact sites: Interaction with porin and adenine nucleotide translocase, role in permeability transition and sensitivity to oxidative damage. Biol Signals Recept 10: 93-111, 2001.

20. Warburg O: On the origin of cancer cells. Science 123: 309-314, 1956.

21. Vander Heiden MG, Cantley LC and Thompson CB: Understanding the Warburg effect: The metabolic requirements of cell proliferation. Science 324: 1029-1033, 2009.

22. Rai Y, Yadav P, Kumari N, Kalra N and Bhatt AN: Hexokinase II inhibition by 3-bromopyruvate sensitizes myeloid leukemic cells K-562 to anti-leukemic drug, daunorubicin. Biosci Rep 39: BSR20190880, 2019.

23. Guo X, Zhang X, Wang T, Xian S and Lu Y: 3-Bromopyruvate and sodium citrate induce apoptosis in human gastric cancer cell line MGC-803 by inhibiting glycolysis and promoting mitochondria-regulated apoptosis pathway. Biochem Biophys Res Commun 475: 37-43, 2016.

24. Luo H, Vong CT, Chen H, Gao Y, Lyu P, Qiu L, Zhao M, Liu Q, Cheng Z, Zou J, et al: Naturally occurring anti-cancer compounds: Shining from Chinese herbal medicine. Chin Med 14: 48, 2019.

25. Guo XX, Li Y, Sun C, Jiang D, Lin YJ, Jin FX, Lee SK and Jin YH: p53-dependent Fas expression is critical for ginsenoside Rh2 triggered caspase- 8 activation in HeLa cells. Protein Cell 5: 224-234, 2014.

26. Li S, Guo W, Gao Y and Liu Y: Ginsenoside Rh2 inhibits growth of glioblastoma multiforme through mTor. Tumour Biol 36: 2607-2612, 2015.

27. Popovich DG and Kitts DD: Structure-function relationship exists for ginsenosides in reducing cell proliferation and inducing apoptosis in the human leukemia (THP-1) cell line. Arch Biochem Biophys 406: 1-8, 2002. 
28. Guo XX, Guo Q, Li Y, Lee SK, Wei XN and Jin YH: Ginsenoside $\mathrm{Rh} 2$ induces human hepatoma cell apoptosisvia bax/bak triggered cytochrome $\mathrm{C}$ release and caspase-9/caspase- 8 activation. Int J Mol Sci 13: 15523-15535, 2012.

29. Choi S, Oh JY and Kim SJ: Ginsenoside Rh2 induces Bcl-2 family proteins-mediated apoptosis in vitro and in xenografts in vivo models. J Cell Biochem 112: 330-340, 2011.

30. Livak KJ and Schmittgen TD: Analysis of relative gene expression data using real-time quantitative PCR and the 2(-Delta Delta C(T)) method. Methods 25: 402-408, 2001.

31. Hu M, Crawford SA, Henstridge DC, Ng IH, Boey EJ, Xu Y, Febbraio MA, Jans DA and Bogoyevitch MA: p32 protein levels are integral to mitochondrial and endoplasmic reticulum morphology, cell metabolism and survival. Biochem J 453: 381-391, 2013.

32. Zhao L, Zhu L and Guo X: Valproic acid attenuates $A \beta$ 25-35-induced neurotoxicity in PC12 cells through suppression of mitochondria-mediated apoptotic pathway. Biomed Pharmacother 106: 77-82, 2018.

33. Liberti MV and Locasale JW: The Warburg effect: How does it benefit cancer cells? Trends Biochem Sci 41: 211-218, 2016

34. Qu C, Zhang S, Wang W, Li M, Wang Y, van der Heijde-Mulder M Shokrollahi E, Hakim MS, Raat NJH, Peppelenbosch MP and Pan Q: Mitochondrial electron transport chain complex III sustains hepatitis $\mathrm{E}$ virus replication and represents an antiviral target. FASEB J 33: 1008-1019, 2019.

35. Pavithra PS, Mehta A and Verma RS: Induction of apoptosis by essential oil from P. Missionis in skin epidermoid cancer cells Phytomedicine 50: 184-195, 2018.

36. Abbaszadeh H, Valizadeh A, Mahdavinia M, Teimoori A, Pipelzadeh MH,Zeidooni L and Alboghobeish S: 3-Bromopyruvate potentiates TRAIL-induced apoptosis in human colon cancer cells through a reactive oxygen species- and caspase-dependent mitochondrial pathway. Can J Physiol Pharmacol 97: 1176-1184, 2019.

37. Pan X, Yan D, Wang D, Wu X, Zhao W, Lu Q and Yan H: Mitochondrion-mediated apoptosis induced by acrylamide is regulated by a balance between Nrf2 antioxidant and MAPK signaling pathways in PC12 cells. Mol Neurobiol 54: 4781-4794, 2017.

38. Camara AKS, Zhou Y, Wen PC, Tajkhorshid E and Kwok WM: Mitochondrial VDAC1: A key gatekeeper as potential therapeutic target. Front Physiol 8: 460, 2017.

39. Xue YN, Yu BB, Li JL, Guo R, Zhang LC, Sun LK, Liu YN and Li Y: Zinc and p53 disrupt mitochondrial binding of HK2 by phosphorylating VDAC1. Exp Cell Res 374: 249-258, 2019.

40. Li M, Shao J, Guo Z, Jin C, Wang L, Wang F, Jia Y, Zhu Z, Zhang Z, Zhang F, et al: Novel mitochondrion-targeting copper(II) complex induces HK2 malfunction and inhibits glycolysis via Drp1-mediating mitophagy in HCC. J Cell Mol Med 24: 3091-3107, 2020

41. Shoshan-Barmatz V, Maldonado EN and Krelin Y: VDAC1 at the crossroads of cell metabolism, apoptosis and cell stress. Cell Stress 1: 11-36, 2017.

42. Ham YM, Lim JH, Na HK, Choi JS, Park BD, Yim H and Lee SK: Ginsenoside-Rh2-induced mitochondrial depolarization and apoptosis are associated with reactive oxygen species- and Ca2+-mediated c-Jun NH2-terminal kinase 1 activation in HeLa cells. J Pharmacol Exp Ther 319: 1276-1285, 2006.

43. Brand MD and Nicholls DG: Assessing mitochondrial dysfunction in cells. Biochem J 435: 297-312, 2011.

44. Bishop T and Brand MD: Processes contributing to metabolic depression in hepatopancreas cells from the snail Helix aspersa. J Exp Biol 203: 3603-3612, 2000.
45. Bustamante MF, Oliveira PG, Garcia-Carbonell R, Croft AP, Smith JM, Serrano RL, Sanchez-Lopez E, Liu X, Kisseleva T, Hay N, et al: Hexokinase 2 as a novel selective metabolic target for rheumatoid arthritis. Ann Rheum Dis 77: 1636-1643, 2018.

46. Park SH, Lee AR, Choi K, Joung S, Yoon JB and Kim S: TOMM20 as a potential therapeutic target of colorectal cancer. BMB Rep 52: 712-717, 2019.

47. Pastorino JG and Hoek JB: Hexokinase II: The integration of energy metabolism and control of apoptosis. Curr Med Chem 10: 1535-1551, 2003

48. Yuan S, Fu Y, Wang X, Shi H, Huang Y, Song X, Li L, Song N and Luo Y: Voltage-dependent anion channel 1 is involved in endostatin-induced endothelial cell apoptosis. FASEB J 22: 2809-2820, 2008.

49. Shoshan-Barmatz V and Ben-Hail D: VDAC, a multi-functional mitochondrial protein as a pharmacological target. Mitochondrion 12: 24-34, 2012.

50. Arbel N, Ben-Hail D and Shoshan-Barmatz V: Mediation of the antiapoptotic activity of $\mathrm{Bcl}-\mathrm{xL}$ protein upon interaction with VDAC1 protein. J Biol Chem 287: 23152-23161, 2012.

51. Zheng Y, Shi Y, Tian C, Jiang C, Jin H, Chen J, Almasan A, Tang $\mathrm{H}$ and Chen Q: Essential role of the voltage-dependent anion channel (VDAC) in mitochondrial permeability transition pore opening and cytochrome $c$ release induced by arsenic trioxide. Oncogene 23: 1239-1247, 2004.

52. Tajeddine N, Galluzzi L, Kepp O, Hangen E, Morselli E, Senovilla L, Araujo N, Pinna G, Larochette N, Zamzami N, et al: Hierarchical involvement of Bak, VDAC1 and Bax in cisplatin-induced cell death. Oncogene 27: 4221-4232, 2008.

53. Li H, Huang N, Zhu W, Wu J, Yang X, Teng W, Tian J, Fang Z, Luo Y, Chen M and Li Y: Modulation the crosstalk between tumor-associated macrophages and non-small cell lung cancer to inhibit tumor migration and invasion by ginsenoside Rh2. BMC Cancer 18: 579, 2018.

54. Li Q, Li B, Dong C, Wang Y and Li Q: 20(S)-Ginsenoside Rh2 suppresses proliferation and migration of hepatocellular carcinoma cells by targeting EZH2 to regulate CDKN2A-2B gene cluster transcription. Eur J Pharmacol 815: 173-180, 2017.

55. Zhang J, Li W, Yuan Q, Zhou J, Zhang J, Cao Y, Fu G and $\mathrm{Hu}$ W: Transcriptome analyses of the anti-proliferative effects of 20(S)-ginsenoside Rh2 on HepG2 cells. Front Pharmacol 10: $1331,2019$.

56. Ma J, Gao G, Lu H, Fang D, Li L, Wei G, Chen A, Yang Y, Zhang $\mathrm{H}$ and Huo J: Reversal effect of ginsenoside $\mathrm{Rh} 2$ on oxaliplatin-resistant colon cancer cells and its mechanism. Exp Ther Med 18: 630-636, 2019.

57. Li B, Zhao J, Wang CZ, Searle J, He TC, Yuan CS and Du W: Ginsenoside Rh2 induces apoptosis and paraptosis-like cell death in colorectal cancer cells through activation of p53. Cancer Lett 301: 185-192, 2011

58. Wang CZ, Zhang B, Song WX, Wang A, Ni M, Luo X, Aung HH, Xie JT, Tong R, He TC and Yuan CS: Steamed American ginseng berry: Ginsenoside analyses and anticancer activities. J Agric Food Chem 54: 9936-9942, 2006.

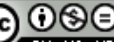

This work is licensed under a Creative Commons Attribution-NonCommercial-NoDerivatives 4.0 International (CC BY-NC-ND 4.0) License. 